\title{
Análise comparativa entre pino de fibra de vidro e núcleo metálico fundido: uma revisão integrativa
}

\author{
Comparative analysis between fiberglass post and cast metal core: an integrative review \\ Análisis comparativo entre poste de fibra de vidrio y núcleo de metal fundido: una revisión \\ integradora
}

Recebido: 19/04/2021 | Revisado: 26/04/2021 | Aceito: 01/05/2021 | Publicado: 15/05/2021

Laryssa Kelly Barbosa Ferro Oliveira

ORCID: https://orcid.org/0000-0001-6967-6461

Universidade Tiradentes de Alagoas, Brasil

E-mail: laryssakellyferro@gmail.com

Savana Ranyella Correia da Silva

ORCID: https://orcid.org/0000-0002-2486-8408

Universidade Tiradentes de Alagoas, Brasil

E-mail: savana1998@hotmail.com

Vanessa Silva de Moura

ORCID: https://orcid.org/0000-0002-3000-6230

Universidade Tiradentes de Alagoas, Brasil

E-mail: vanessamoura37@gmail.com

Anne Myrelle da Cruz Andrade

ORCID: https://orcid.org/0000-0003-2637-7271

Universidade Tiradentes de Alagoas, Brasil

E-mail: annemyrelle@hotmail.com

Lucas Mariz de Menezes Torres

ORCID: https://orcid.org/0000-0001-7543-7715

Universidade Tiradentes de Alagoas, Brasil

E-mail: lmarizdemenezes@gmail.com

Marielly dos Anjos Ferreira da Silva

ORCID: https://orcid.org/0000-0001-8599-6385

Universidade Tiradentes de Alagoas, Brasil

E-mail: anjosmarielly@gmail.com

Luana Ranna Silva Santos

ORCID: https://orcid.org/0000-0002-2361-633X

Universidade Tiradentes de Alagoas, Brasil

E-mail: luanaranna2014@gmail.com

Daniel Alves da Silva

ORCID: https://orcid.org/0000-0001-9938-2165

Universidade Tiradentes de Alagoas, Brasil

E-mail: odanielalves@outlook.com

Júlia Araújo Gomes

ORCID: https://orcid.org/0000-0003-1323-5071

Universidade Tiradentes de Alagoas, Brasil E-mail: juaraujogomes@hotmail.com

Emillianno de Gusmão Gonçalves

ORCID: https://orcid.org/0000-0002-2248-5016

Centro de Estudos Superiores de Maceió, Brasil

E-mail: emillianno@hotmail.com

\begin{abstract}
Resumo
Os pinos de fibra de vidro ou núcleos metálicos fundidos são utilizados na odontologia como retentores intrarradiculares e tem como principal intuito repor a estrutura dental que foi perdida, além de facilitar o suporte e a retenção da coroa. A revisão teve por objetivo analisar a literatura em relação à comparação entre os pinos de fibra e os núcleos metálicos fundidos. Foi desenvolvida uma revisão integrativa da literatura nas bases de dados SciELO e LILACS, utilizando os descritores: "Reabilitação bucal", "Pinos dentários" e "Técnica para retentor intrarradicular" individualmente e em seguida o cruzamento com o operador booleano AND. Os critérios de inclusão utilizados na seleção dos artigos foram: artigos publicados em português disponíveis na íntegra que retratassem o problema pesquisado, artigos publicados e indexados nos referidos bancos de dados nos últimos quinze anos (2005 a 2020). Foram encontrados 20 artigos e a partir deles verificado que o uso de núcleos metálicos fundidos está diminuindo, devido ao sucesso dos pinos estéticos. A fratura radicular é menor em dentes restaurados com pinos de fibra de vidro devido à melhor distribuição de forças. Apesar disso, é fato que tanto os núcleos metálicos fundidos quanto os pinos de fibra de vidro apresentam bons resultados quando utilizados corretamente, mas os pinos de fibra de vidro apresentam
\end{abstract}


maior resistência à fratura. Mais estudos podem ser feitos com diversas marcas em uso no mercado, para guiar melhor o cirurgião-dentista sobre os materiais em uso.

Palavras-chave: Pinos dentários; Reabilitação bucal; Técnica para retentor intrarradicular.

\begin{abstract}
Fiberglass pins or fused metal cores are used in dentistry as intraradicular retainers and have the main purpose of replacing the lost dental structure, in addition to facilitating the support and retention of the crown. The purpose of the review was to analyze the literature in relation to the comparison between fiber pins and fused metal cores. An integrative literature review was developed in the SciELO and LILACS databases, using the descriptors: "Mouth Rehabilitation", "Dental pins" and "Post and Core Technique" individually and then the crossing with the Boolean operator AND. The inclusion criteria used in the selection of articles were: articles published in Portuguese available in full that portrayed the problem researched, articles published and indexed in the referred databases in the last fifteen years (2005 to 2020). Twenty articles were found and from them it was verified that the use of fused metal cores is decreasing, due to the success of aesthetic pins. Root fracture is less in teeth restored with fiberglass pins due to the better distribution of forces. Despite this, it is a fact that both fused metal cores and fiberglass pins have good results when used correctly, but fiberglass pins have greater resistance to fracture. Further studies can be done with several brands in use in the market, to better guide the dentist about the materials in use.
\end{abstract}

Keywords: Dental pins; Mouth rehabilitation; Post and core technique.

\title{
Resumen
}

Los pines de fibra de vidrio o núcleos de metal fundido se utilizan em odontología como retenedores intrarradiculares y tienen el propósito principal de reemplazar la estructura dental perdida, además de facilitar el soporte y retención de la corona. El propósito de la revisión fue analizar la literatura em relación a la comparación entre pines de fibra y núcleos de metal fundido. Se desarrolló una revisión integradora de la literatura em las bases de datos SciELO y LILACS, utilizando los descriptores: "Rehabilitación Bucal", "Pins Dentales" y "Técnica de Perno Muñón” individualmente y luego el cruce conel operador booleano AND. Los criterios de inclusión utilizados em la selección de artículos fueron: artículos publicados em português disponibles em su totalidad que retrataran el problema investigado, artículos publicados e indexados em las referidas bases de datosenlos últimos quinceaños (2005 a 2020). Se encontraron veinte artículos y de ellos se comprobó que el uso de núcleos de metal fundido está disminuyendo, debido al éxito de los pines estéticos. La fractura de laraíz es menor em los dientes restaurados conpasadores de fibra de vidrio debido a la mejor distribución de fuerzas. A pesar de esto, es unhecho que tanto los núcleos de metal fundido como los pines de fibra de vidrio tienen buenos resultados cuando se usan correctamente, pero los pines de fibra de vidrio tienen una mayor resistencia a la fractura. Se pueden realizar más estudios con varias marcas en uso en el mercado, para orientar mejor al dentista sobre los materiales en uso.

Palabras clave: Pins dentales; Rehabilitación bucal; Técnica de perno muñón.

\section{Introdução}

Dentes que perderam grande parte de sua estrutura coronária necessitam de dispositivos intrarradiculares para alcançar uma retenção desejada do material restaurador, devolvendo estética e função ao paciente (Sigemori et al., 2012). Os pinos de fibra de vidro e núcleos metálicos fundidos são utilizados na odontologia como retentores intrarradiculares, tendo como principal intuito repor a estrutura dental que foi perdida, além de facilitar o suporte e a retenção da coroa (Moro, Agostinho \& Matsumoto, 2005). É necessário que o pino escolhido pelo cirurgião-dentista e utilizado durante o tratamento, consiga suportar o estresse, seja biocompatível, tenha uma boa resposta quando associado a outros materiais restauradores e seja de fácil colocação e remoção. Além disso, os tecidos de suporte dentário devem apresentar saúde favorável (Soares \& Sant'ana, 2018).

Apesar da menor resistência a fraturas, dentes que são tratados endodonticamente, com o passar dos anos estão apresentando evolução significativa em relação às técnicas de restauração e reconstrução (Sigemori et al., 2012). O núcleo metálico é uma das técnicas de reabilitação em dentes que passaram por terapia pulpar e apresentam uma perda elevada de estrutura dentária. É um núcleo produzido com ligas metálicas como níquel-cromo e bastante indicado por apresentar adaptação favorável ao conduto radicular e também uma boa resistência (Sá, Akaki \& Sá, 2010).

O emprego de pinos estéticos compostos por fibra de vidro tem sido cada vez mais estudado e os benefícios biomecânicos, além da possibilidade adesiva, tornam viável seu uso em várias situações clínicas. Estudos mostram que a 
perfeita adaptação às paredes do canal radicular é importante para melhorar as propriedades do conjunto dente-pino (Oliveira et al., 2012). Aspectos importantes a serem considerados são anatomia intrarradicular, a fricção/adaptação do pino no interior do canal radicular e a dificuldade de acesso nas regiões mais profundas do conduto, controle de umidade e fator-C (Marques et al., 2014).

Núcleos metálicos fundidos apresentam boas propriedades mecânicas, mas possuem uma estética desfavorável e necessitam de um tempo clínico mais elevado para serem confeccionados, então, por conta disso, estão sendo cada dia menos indicados (Sá, Akaki \& Sá, 2010). Pinos de fibra de vidro e fibra de carbono além de possuírem estética favorável, têm também efetivas propriedades físicas e mecânicas e são capazes de proporcionarem aproveitamento mais prudente do remanescente dentário, resultando num tratamento mais conservador, proporcionando uma melhor recuperação de dentes que foram danificados extensamente (Prado et al., 2014). É importante ressaltar que a seleção do retentor mais adequado para cada situação clínica pode interferir diretamente na longevidade do tratamento restaurador (Mazaro et al., 2014).

O objetivo deste estudo é analisar a literatura comparando pinos de fibra e os núcleos metálicos fundidos, ressaltando suas características, indicações, vantagens, desvantagens e uso na odontologia.

\section{Material e Métodos}

Este estudo trata-se de uma revisão integrativa da literatura, baseada nas seis etapas descritas por Mendes et al., (2008): pergunta norteadora da pesquisa, busca na literatura, categorização dos estudos, avaliação dos estudos, interpretação dos resultados e síntese do conhecimento. Na qual a questão norteadora para pesquisa foi: "Tratando-se de pino de fibra de vidro e núcleo metálico fundido, qual a melhor opção de uso na odontologia?".

Para o levantamento dos artigos na literatura utilizou-se duas bases de dados, Scientific Electronic Library Online (SciELO) e Literatura Latino-americana e do Caribe em Ciências da Saúde (LILACS) e livros atuais que retratassem o tema proposto.

Para o levantamento dos artigos foram utilizados como descritores: "Reabilitação bucal", "Pinos dentários" e "Técnica para retentor intrarradicular". O levantamento bibliográfico foi realizado em Maio de 2020. Inicialmente, a busca pelos descritores foi feita individualmente e em seguida foram realizados os cruzamentos utilizando o operador booleano AND. Os critérios de inclusão definidos para a seleção dos artigos foram: artigos publicados em português disponíveis na íntegra que retratassem o problema pesquisado, artigos publicados e indexados nos referidos bancos de dados nos últimos quinze anos (2005 a 2020), os critérios de exclusão seguiram de: artigos publicados antes de 2005, em outros idiomas, além do português, monografias e trabalhos de conclusão de curso.

\section{Resultados}

A amostra final desta revisão foi constituída por 23 artigos científicos, dos quais 3 se repetiram, restando apenas 20 artigos, selecionadas através dos critérios de inclusão previamente estabelecidos. Destes, 15 foram encontrados na base de dados LILACS e os demais na base de dados SCIELO.

O quadro a seguir expõe os artigos selecionados nas bases de dados. 
Quadro 1. Artigos incluídos na revisão integrativa.

\begin{tabular}{|c|c|c|c|c|}
\hline Título & Autores & Ano & Delineamento & Desfecho \\
\hline $\begin{array}{l}\text { Retenção de pinos pré-fabricados } \\
\text { e núcleos metálicos fundidos com } \\
\text { cimento resinoso e fosfato de } \\
\text { zinco. }\end{array}$ & Shiozawa, L. J.et al. & 2005 & $\begin{array}{l}\text { Estudo } \\
\text { comparativo }\end{array}$ & $\begin{array}{l}\text { A cimentação realizada com cimento resinoso } \\
\text { e o fosfato de zinco apresentam capacidade } \\
\text { retentiva e resistência à remoção por tração } \\
\text { maior. }\end{array}$ \\
\hline $\begin{array}{l}\text { Núcleos Metálicos Fundidos X } \\
\text { Pinos Pré-Fabricados. }\end{array}$ & $\begin{array}{l}\text { Moro, M., } \\
\text { Agostinho, A. M. \& } \\
\text { Matsumoto, W. }\end{array}$ & 2005 & $\begin{array}{l}\text { Revisão de } \\
\text { literatura }\end{array}$ & $\begin{array}{l}\text { Ainda há indicação específica para cada um } \\
\text { dos pinos pré-fabricados. Os núcleos } \\
\text { metálicos fundidos são muito empregados e } \\
\text { quando bem indicados, permitem bons } \\
\text { resultados clínicos. }\end{array}$ \\
\hline $\begin{array}{l}\text { Comparação da resistência à } \\
\text { tração entre pinos metálicos } \\
\text { (ni/cr) e de fibra de vidro } \\
\text { cimentados com cimento } \\
\text { resinoso. }\end{array}$ & Silva, R. V. C.et al. & 2009 & $\begin{array}{l}\text { Estudo } \\
\text { comparativo }\end{array}$ & $\begin{array}{l}\text { O núcleo metálico fundido e o pino de fibra de } \\
\text { vidro mostram valores parecidos de } \\
\text { resistência à tração, quando estes são } \\
\text { cimentados com cimentos resinosos. }\end{array}$ \\
\hline $\begin{array}{l}\text { Pinos intrarradiculares estéticos - } \\
\text { caso clínico. }\end{array}$ & $\begin{array}{l}\text { Franco, A. P. G. O. } \\
\text { et al. }\end{array}$ & 2009 & Relato de caso & $\begin{array}{l}\text { Pinos intrarradiculares estéticos têm se } \\
\text { mostrado eficientes quanto à retenção do } \\
\text { material restaurador coronário de dentes } \\
\text { mutilados e na distribuição das cargas } \\
\text { mastigatórias. }\end{array}$ \\
\hline $\begin{array}{l}\text { Pinos estéticos: Qual o melhor } \\
\text { sistema? }\end{array}$ & $\begin{array}{l}\text { Sá, T. C. M., Akaki, } \\
\text { E. \& Sá, J. C. M. }\end{array}$ & 2010 & $\begin{array}{l}\text { Revisão de } \\
\text { literatura }\end{array}$ & $\begin{array}{l}\text { O uso de pinos fundidos vem diminuindo, } \\
\text { devido ao sucesso dos pinos estéticos. A } \\
\text { fratura radicular é menor em dentes } \\
\text { restaurados com fibra de vidro se comparado } \\
\text { aos com pinos cerâmicos e metálicos } \\
\text { fundidos, devido à melhor distribuição de } \\
\text { forças. }\end{array}$ \\
\hline $\begin{array}{l}\text { Remoção de retentores metálicos } \\
\text { intrarradiculares com o saca- } \\
\text { pinos M\&V: relato de casos } \\
\text { clínicos. }\end{array}$ & $\begin{array}{l}\text { Pantoja, C. A. M. S. } \\
\text { et al. }\end{array}$ & 2011 & Relato de caso & $\begin{array}{l}\text { Saca-pino M\&V® é eficiente para a remoção } \\
\text { de retentores metálicos intrarradiculares e } \\
\text { associado a outros recursos torna o } \\
\text { procedimento mais rápido, fácil e previsível. }\end{array}$ \\
\hline $\begin{array}{l}\text { Reforço intrarradicular de raízes } \\
\text { debilitadas. }\end{array}$ & $\begin{array}{l}\text { Sigemori, R. M. et } \\
\text { al. }\end{array}$ & 2012 & $\begin{array}{l}\text { Revisão de } \\
\text { literatura }\end{array}$ & $\begin{array}{l}\text { Estrutura dental e as paredes do canal ajudam } \\
\text { na resistência à fratura, porém a instalação de } \\
\text { pinos pré-fabricados enfraquecem os dentes, } \\
\text { tomando-os mais susceptível a fraturas. }\end{array}$ \\
\hline $\begin{array}{l}\text { Distribuição das tensões } \\
\text { relacionadas ao uso de retentores } \\
\text { em dentes tratados } \\
\text { endodonticamente utilizando o } \\
\text { método dos finitos. }\end{array}$ & Oliveira, H. E. et al. & 2012 & $\begin{array}{l}\text { Revisão de } \\
\text { literatura }\end{array}$ & $\begin{array}{l}\text { Pinos intrarradiculares metálicos de titânio } \\
\text { geram estresse no sistema dente-periodonto, } \\
\text { sendo o menos indicado. O mais indicado foi } \\
\text { o pino metálico fundido, }\end{array}$ \\
\hline $\begin{array}{l}\text { Influência da espessura do filme } \\
\text { de cimento na resistência de } \\
\text { união de pinos reforçados por } \\
\text { fibras cimentados em canais } \\
\text { radiculares. }\end{array}$ & Prado, N. A. S. et al. & 2013 & $\begin{array}{l}\text { Revisão de } \\
\text { literatura }\end{array}$ & $\begin{array}{l}\text { O devido assunto deve continuar sendo } \\
\text { investigado, com o intuito de um protocolo } \\
\text { clínico mais confiável, que promova menos } \\
\text { desgaste de estrutura do elemento dental } \\
\text { durante o preparo do conduto, para que não } \\
\text { ocorra prejuízo na resistência adesiva. }\end{array}$ \\
\hline $\begin{array}{l}\text { Retentores Intrarradiculares: } \\
\text { Revisão da Literatura. }\end{array}$ & $\begin{array}{l}\text { Prado, M. A. A. et } \\
\text { al. }\end{array}$ & 2014 & $\begin{array}{l}\text { Revisão de } \\
\text { literatura }\end{array}$ & $\begin{array}{l}\text { Os núcleos metálicos fundidos ainda são } \\
\text { bastante utilizados, porém, a indicação dos } \\
\text { pinos pré-fabricados flexíveis vem ganhando } \\
\text { destaque, pois suas propriedades mecânicas } \\
\text { são mais favoráveis na restauração. }\end{array}$ \\
\hline
\end{tabular}




\begin{tabular}{|c|c|c|c|c|}
\hline $\begin{array}{l}\text { Avaliação da resistência de união } \\
\text { entre dentina e pinos de fibra de } \\
\text { vidro utilizando três diferentes } \\
\text { técnicas de cimentação. }\end{array}$ & Marques, V. F. et al. & 2014 & $\begin{array}{l}\text { Estudo } \\
\text { comparativo }\end{array}$ & $\begin{array}{l}\text { O sucesso clínico da cimentação de pinos } \\
\text { intrarradiculares, com materiais resinosos, } \\
\text { deve-se, predominantemente, à retenção por } \\
\text { atrito e não apenas à ligação adesiva } \\
\text { propriamente dita. }\end{array}$ \\
\hline $\begin{array}{l}\text { Avaliação dos fatores críticos } \\
\text { para seleção e aplicação clínica } \\
\text { dos pinos de fibra: relato de caso. }\end{array}$ & $\begin{array}{l}\text { Mazaro, J. V. Q. et } \\
\text { al. }\end{array}$ & 2014 & Relato de caso & $\begin{array}{l}\text { Os pinos de fibra apresentam-se como uma } \\
\text { alternativa para reabilitações estéticas em } \\
\text { região anterior. }\end{array}$ \\
\hline $\begin{array}{l}\text { Efeitos do reembasamento sobre } \\
\text { a resistência ao cisalhamento por } \\
\text { extrusão (push-out) de pinos de } \\
\text { fibra de vidro. }\end{array}$ & $\begin{array}{l}\text { Andrioli, A. R. V. et } \\
\text { al. }\end{array}$ & 2016 & $\begin{array}{l}\text { Estudo } \\
\text { comparativo }\end{array}$ & $\begin{array}{l}\text { A utilização dos pinos de fibra de vidro é } \\
\text { essencial para que se tenha sucesso no } \\
\text { tratamento restaurador, os pinos não } \\
\text { reembasavel tem maior resistência ao } \\
\text { cisalhamento. }\end{array}$ \\
\hline $\begin{array}{l}\text { Análise comparativa da } \\
\text { resistência de união de um } \\
\text { cimento convencional e um } \\
\text { cimento autoadesivo após } \\
\text { diferentes tratamentos na } \\
\text { superfície de pinos de fibra de } \\
\text { vidro. }\end{array}$ & Marques, J. N.et al. & 2016 & $\begin{array}{l}\text { Estudo } \\
\text { comparativo }\end{array}$ & $\begin{array}{l}\text { O cimento convencional e o autoadesivo } \\
\text { apresentam uma adesão satisfatória e } \\
\text { semelhante entre a camada de dentina e o pino } \\
\text { de fibra de vidro. }\end{array}$ \\
\hline $\begin{array}{l}\text { Pino de fibra de vidro anatômico: } \\
\text { relato de caso. }\end{array}$ & $\begin{array}{l}\text { Ferreira, M. B. C. et } \\
\text { al. }\end{array}$ & 2018 & Relato de caso & $\begin{array}{l}\text { Os pinos de fibra anatômicos apresentam } \\
\text { vantagem em comparação aos pré-fabricados, } \\
\text { pois se adequam melhor ao canal radicular de } \\
\text { elementos dentais que possuem tratamento } \\
\text { endodôntico. }\end{array}$ \\
\hline $\begin{array}{l}\text { Estudo Comparativo entre Pino } \\
\text { de Fibra de Vidro e Pino } \\
\text { Metálico Fundido: Uma Revisão } \\
\text { de Literatura. }\end{array}$ & $\begin{array}{l}\text { Soares, D. N. S. \& } \\
\text { Sant'ana L. L. P. }\end{array}$ & 2018 & $\begin{array}{l}\text { Revisão de } \\
\text { literatura }\end{array}$ & $\begin{array}{l}\text { Fatores como a dentina remanescente e a } \\
\text { quantidade de estrutura dental restante } \\
\text { influenciam diretamente na escolha no } \\
\text { retentor intrarradicular. }\end{array}$ \\
\hline $\begin{array}{l}\text { Resistência à fratura de dentes } \\
\text { tratados endodonticamente } \\
\text { reforçados com pinos } \\
\text { customizados de fibra de vidro e } \\
\text { carbono }\end{array}$ & Carvalho, T. F. et al. & 2019 & $\begin{array}{l}\text { Estudo } \\
\text { comparativo }\end{array}$ & $\begin{array}{l}\text { Quatro tipos de pinos comparados quanto a } \\
\text { resistência à fratura, ficando evidente que o } \\
\text { uso de pinos customizados é capaz de reduzir } \\
\text { a incidência de fraturas, já a ausência do pino } \\
\text { intrarradicular é capaz de causar fraturas do } \\
\text { tipo irreparáveis. }\end{array}$ \\
\hline $\begin{array}{l}\text { Avaliação do efeito da } \\
\text { clorexidina e hipoclorito de sódio } \\
\text { na cimentação adesiva de pinos } \\
\text { de fibra - estudo in vitro. }\end{array}$ & Conte, T.et al. & 2019 & Estudo de caso & $\begin{array}{l}\text { Tanto a clorexidina como o hipoclorito de } \\
\text { sódio apresentam resistência de união adesiva } \\
\text { à dentina na fixação dos pinos de fibra de } \\
\text { vidro. }\end{array}$ \\
\hline $\begin{array}{l}\text { Potencial antimicrobiano de } \\
\text { diferentes retentores } \\
\text { intrarradiculares frente } \\
\text { a Enterococcusfaecalis: uma } \\
\text { avaliação in vitro. }\end{array}$ & $\begin{array}{l}\text { Finger, N. H.; } \\
\text { Paulus, M.; Gazzoni, } \\
\text { A. F. }\end{array}$ & 2019 & Estudo de caso & $\begin{array}{l}\text { Retentores intrarradiculares dos grupos G3- } \\
\mathrm{Ni}-\mathrm{Cr} \text { e } \mathrm{G} 4-\mathrm{Cu}-\mathrm{Al} \text { apresentou efeito } \\
\text { antimicrobiano maior frente ao } \\
\text { microrganismo testado. Confirmando assim a } \\
\text { alternativa de usa-los nas etapas protéticas na } \\
\text { realização da restauração. }\end{array}$ \\
\hline $\begin{array}{l}\text { Desgaste dentinário seletivo } \\
\text { associado à pino de fibra de } \\
\text { vidro: relato de caso }\end{array}$ & $\begin{array}{l}\text { Pessoa, V. L. P. et } \\
\text { al. }\end{array}$ & 2019 & Relato de caso & $\begin{array}{l}\text { Manchas dentinária intrínsecas podem ocorrer } \\
\text { por diversos motivos como hemorragia } \\
\text { intracoronária e medicamentos de uso } \\
\text { intracanal, causando comprometimento } \\
\text { estético - funcional do sorriso e os pinos } \\
\text { quando usados de forma correta devolvem } \\
\text { favoravelmente à estética e autoestima dos } \\
\text { pacientes. }\end{array}$ \\
\hline
\end{tabular}


Fonte: Autores.

No quadro acima é possível verificar que os artigos foram publicados entre 2005 a 2019, nos quais20 foram selecionados e organizados por meio de fichamento, em ordem numérica crescente, tendo dois (2) artigos de estudo de caso, cinco (5) artigos de relato de caso, seis (6) artigos de estudo comparativo e sete (7) artigos de revisão de literatura, possibilitando assim a aproximação inicial com o tema. Em seguida, os artigos foram submetidos à leitura na íntegra, tendo como finalidade proporcionar uma melhor busca dos dados apresentados pelos artigos, além de visar uma realização de análise interpretativa.

\section{Discussão}

A perda de estrutura dentária causada por lesão cariosa, aliada ao tratamento endodôntico, pode comprometer a resistência do elemento dental, assim a restauração desses elementos dentais torna-se um desafio para a odontologia restauradora, dando à carência de remanescente dental. Quando a estrutura dentinária está insatisfatória, dispõe-se do uso de pinos intrarradiculares para elevar a resistência à fratura, propiciando uma reabilitação restauradora e assim alcançando uma maior longevidade ao dente (Oliveira et al., 2012, Moro, Agostinho \& Matsumoto, 2005)

Os pinos intrarradiculares diferem-se pela forma anatômica, configuração superficial e composição do material, entretanto detém da mesma função de reforçar o remanescente dentinário. Devido a essa grande variedade é de fundamental importância que o cirurgião dentista domine o conhecimento sobre a classificação desse material, para facilitar a seleção diante dos casos clínicos (Soares \& Sant’ana, 2018).

A partir da forma anatômica podem ser classificados como cilíndricas ou cônicas; De acordo com a configuração superficial podem ser classificados como lisa, serrilhada ou rosqueáveis; Já os materiais de confecção são classificados como metálicas (titânio ou aço inoxidável) ou não metálicas, sendo subdivididas em "não estéticos" (fibras de carbono) ou "estéticos" (fibras de vidro, fibras de quartzo, fibras de carbono com quartzo e dióxido de zircônio) (Franco et al., 2009).

A indicação ou não dos pinos intrarradiculares é baseado em parâmetros como: posição do elemento dental no arco; oclusão; função do dente na arcada; quantidade de remanescente dentário e a morfologia do canal. Estes retentores intrarradiculares estão indicados para reforçarem elementos dentais com tratamento endodôntico, de modo que, retenham e restaurarem a estrutura destruída, além de evitar futuras fraturas (Mazaro et al., 2014). Em casos de insucesso neste tratamento e havendo necessidade de retratamento do sistema de canais radiculares, o cirurgião-dentista pode sentir dificuldade durante a remoção dos retentores intrarradiculares levando ao risco de fraturas no remanescente radicular. Nesses casos o profissional deve lançar mão de técnicas mais eficientes para uma remoção com maior segurança, como o Saca-pinos, que segundo Pantoja et al., (2011) é um aparelho que pode ser usado isolado ou associado a outras técnicas e funciona exercendo uma força de tração para remover o retentor intrarradicular.

Prado et al., (2013) e Soares e Sant'ana (2018) ressaltam sobre as diferentes opções de retentores intrarradiculares para dentes que possuem tratamento endodôntico, dentre eles pode-se destacar os núcleos metálicos fundidos e os pinos de fibra de vidro. O objetivo dos núcleos metálicos fundidos é repor a perda de estrutura perdida do dente, além disso, facilita o suporte e retenção da coroa dental. Possuem em sua composição ligas metálicas distintas: níquel-cromo, prata-paládio e cobrealumínio, são algumas delas. Podem ser indicados devido a sua boa resistência e à sua adequada adaptação nos condutos radiculares, mas, sua cor prateada e seu tempo elevado de confecção, são desvantajosos. Os pinos de fibra de vidro podem servir como substitutos aos pinos metálicos, pois favorece a estética, devido a sua coloração similar ao da estrutura dentária, e, quando utilizados precisam de um menor desgaste de dentina intrarradicular (Prado et al., 2013; Soares \& Sant'ana, 2018). 
Através de uma avaliação in vitro, Finger, Paulus e Gazzoni (2019), evidenciaram que os retentores intrarradiculares agregados aos grupos G3-Ni-Cr e G4-Cu-Al possuem um maior efeito antimicrobiano frente ao microrganismo Enterococcus faecalis. Sendo assim, confirmam que é uma boa alternativa usá-los em etapas protéticas na realização da restauração.

\section{Núcleos metálicos}

O núcleo metálico fundido é considerado uma das técnicas mais antigas aplicadas na odontologia por preencher melhor o propósito no qual é destinado. São núcleos muito resistentes, versáteis e permitem uma adequada adaptação no canal radicular, tem também como vantagem o baixo custo, não necessita de técnica ou cimentos especiais para fixação, grande experiência clínica nas várias décadas em que já são empregadas, além de excelente radiopacidade (Moro, Agostinho \& Matsumoto, 2005). Seu uso é indicado em situações no qual os pinos pré-fabricados não se adequem as paredes dos condutos radiculares, em mudança na inclinação dentária e em grandes perdas de estrutura coronária (Prado et al.,2014; Mazaro et al.,2014).

É de extrema importância que o núcleo metálico fundido apresente extensão de aproximadamente $2 / 3$ do comprimento radicular, além disso, o mesmo deve permanecer no mínimo a 4,0 mm de material para obturação no ápice do elemento dental para que ocorra uma adequada cimentação do núcleo metálico fundido (Prado et al., 2014).Essas medidas são essenciais, pois se o comprimento do núcleo inserido ao dente for superior, pode haver enfraquecimento da raiz devido à supressão da dentina, que provavelmente ocasionará numa fratura radicular durante os episódios de cargas mastigatórias (Prado et al.,2013).

Segundo Moro, Agostinho e Matsumoto (2005) Na tentativa de prover as deficiências dos núcleos metálicos fundidos, como necessidade de fase laboratorial e a estética desfavorável, surgiram os pinos pré-fabricados. Entre as diversas classes de pinos pré-fabricados existentes no mercado, tendo características e indicações específicas para cada tipo, estes apresentam vantagens de fácil e rápida instalação; dispensam moldagem; tem baixo custo e preparo conservador; disponível em diversas formas, tamanho e materiais, porém segundo Sá et al., (2010) os pinos metálicos pré-fabricados exibem potencial de fraturas radiculares em médio e curto prazo, além da desfavorável coloração do material.

Outras desvantagens também são observadas como enfraquecimento da raiz; falta de retenção do agente de cimentação, dificuldade de remoção; tempo de trabalho estendido, estética desfavorável, probabilidade de sofrerem corrosão, módulo de elasticidade sendo superior ao da dentina, além de não serem adesivos (Sá, Akaki \& Sá, 2010; Moro, Agostinho \& Matsumoto, 2005).

\section{Pinos de fibra}

Os pinos intrarradiculares têm como objetivo promover a retenção da restauração de dentes com perda severa da estrutura coronária e que foram tratados endodonticamente. Por muito tempo os pinos metálicos eram utilizados com essa finalidade, mas com o aumento das exigências estéticas na odontologia aos poucos começaram a ser introduzidas no mercado os pinos não-metálicos, entre eles o pinos reforçados por fibras (Prado et al.,2013).

Pinos reforçados por fibra, especialmente o pino de fibra de vidro são materiais que em sua composição apresentam fibras longitudinais de vidro e quanto maior a quantidade de fibras, maior a resistência e a rigidez. Essas fibras geralmente são combinadas com uma matriz resistente de resina composta. (Prado et al., 2013; Soares \& Sant'ana, 2018). Pessoa et al., (2019) relatam que quando o desgaste dentinário acontece de forma seletiva e está associado à restauração utilizando resina composta juntamente com o pino de fibra de vidro, há favorecimento na estética e função do elemento dentário e autoestima do paciente.

São indicados para reabilitação de elementos dentais com tratamento endodôntico e que apresentam cerca de metade do remanescente coronário, sendo contraindicado em elementos dentais que possuem canais amplos. Uma das vantagens que se destacam é o módulo de elasticidade perto ao da dentina, fazendo com que as forças da mastigação sejam absorvidas, 
protegendo assim o remanescente radicular do elemento dental, ademais contam com valores altos de adesão as resinas odontológicas. Caso seja necessário um retratamento endodôntico, estes elementos possuem uma maior facilidade para remoção, possuem resistência à corrosão e admitem um preparo mais conservador do elemento dentário. Sua utilização não necessita de fases em laboratórios, sendo assim, há economia na duração do tempo clínico e consequentemente nos custos (Prado et al., 2014).

Pinos de fibra de carbono também são opções de bastante relevância e surgiram no mercado odontológico em meados dos anos 90, como possíveis substitutos dos pinos metálicos. São constituídos por cerca de $64 \%$ de fibra de carbono e apresentam $8 \mu \mathrm{m}$ de diâmetro que ficam dispostos longitudinalmente, evolvidos numa matriz de resina epóxica. Como vantagens, esse pino de fibra apresenta boa flexibilidade, biocompatibilidade e aderência química em relação às resinas de uso odontológico, não necessitam de qualquer tratamento de superfície, possuem resistência à corrosão, resistência à fadiga, características mecânicas semelhantes às da dentina e facilidade de remoção. Como desvantagem pode ter falhas adesivas, pois é um material flexível (Moro, Agostinho \& Matsumoto, 2005).

Entre todos os retentores utilizados, os pinos de fibra são considerados os preferidos entre os cirurgiões dentistas, pois possuem mais praticidade, são econômicos, com elevada estética e translucidez, favorece a cimentação adesiva, seu módulo de elasticidade se assemelha a estrutura dentária e apresentam melhor distribuição de tensões ao longo da raiz e por isso são muito aproveitados em dentes anteriores (Mazaro et al., 2014).

\section{Potencial de resistência à fratura}

O sistema de retenção intrarradicular tem como objetivo prover resistência à fratura do remanescente dentinário e fornecer retenção para o material restaurador (Sigemori et al., 2012), Entretanto, dentes que foram submetidos a tratamento endodôntico estão mais suscetíveis à fratura por conta de redução da umidade dentinária (Prado et al., 2013), sendo assim, há necessidade de manutenção da estrutura dentária, avaliando sempre a resistência do elemento dental. À vista disso, a estrutura dentinária saudável deve ser preservada durante a inserção de qualquer meio auxiliar de retenção intrarradicular, em razão disso a instalação de núcleos metálicos fundidos ou pinos pré-fabricados enfraquecem a estrutura dental, assim deixando-a mais suscetível a fraturas radiculares verticais. Diante disso, o uso de pinos deve ser indicado exclusivamente para retenção do material restaurador e não como reforço para dentes com tratamento endodôntico, pois sempre se remove dentina sadia para a instalação desses meios de retenção (Sigemori et al., 2012; Andrioli et al., 2016).

Os pinos reforçados por fibras são formados por fibras inorgânicas de carbono, vidro ou quartzo, embebidas em matriz de resina epóxi ou de metacrilato, para permitir uma melhor distribuição das tensões pelo pino. As fibras aumentam a rigidez dos pinos, assim uma maior densidade de fibras aumenta sua resistência à fratura (Ferreira et al., 2018), além de, segundo Carvalho et al., (2018) o módulo de elasticidade entre dentina, pino de fibra de vidro e resina composta serem muito semelhantes, fazendo com que as tensões sejam distribuídas de forma mais homogênea e a interface dentina/pino seja preservada, reduzindo o risco de fratura, permitindo o que leva a uma melhor distribuição de tensões e preservação da interface dentina/pino, melhorando assim a resistência à fratura. Quando os pinos de fibra de vidro são utilizados, a transferência de tensão para estruturas radiculares é menor, que possivelmente reduzem o índice de fraturas, principalmente em raízes fragilizadas (Prado et al., 2013).

Um aumento na concentração de tensão no ápice radicular pode ser causado pelos núcleos metálicos fundidos, visto que estes retentores dispõem de um módulo de elasticidade mais elevado que o da dentina. Consequentemente as cargas de mastigação são passadas para o núcleo através da coroa protética, e logo depois são transferidas aos tecidos dos elementos dentais, sendo adiante dissipadas como deformação elástica. Caso o valor da tensão seja mais elevado que o limite elástico e a força de coesão tecidual a raiz do elemento dentário poderá sofrer uma fratura de curto e médio prazo (Prado et al., 2013). 


\section{Resistência de união}

Segundo Silva et al., (2009) deixam claro que tanto o núcleo metálico fundido quanto o pino de fibra de vidro expõem valores similares de resistência à tração, quando estes são cimentados com cimentos resinosos. Os retentores intrarradiculares são responsáveis tanto pela retenção da restauração final, quanto pela proteção do remanescente radicular. Para que se tenha uma retenção adequada da restauração, o retentor deve ser de no mínimo $2 / 3$ do comprimento da raiz do dente e ser maior ou igual à coroa futura (Shiozawa et al., 2005).

Segundo Prado et al., (2013) quanto maior espessura do filme de cimento menor a resistência adesiva. Quando o pino está precisamente adaptado ao conduto preparado, a resistência adesiva diminui. Marques et al., (2014) relatam que quanto mais íntimo o contato entre o cimento resinoso e as paredes do canal, maior será a retenção. Shiozawa et al., (2005) completam falando que quando ocorre cimentação com cimento resinoso e fosfato de zinco, ocorre uma maior capacidade de retenção e resistência a tração aumentada nos retentores intrarradiculares.

Os agentes cimentantes utilizados para execução do tratamento quer que seja o cimento convencional ou o autoadesivo apresentaram uma adesão satisfatória e semelhante entre opino de fibra de vidro e a dentina, além de apresentarem que os valores de resistência de união (RU) semelhantes foram exibidos e achados no grupo de jateamento com óxido de alumínio (Marques et al., 2016).

O agente silano não aumenta a resistência adesiva entre pinos reforçados por fibras e cimento resinoso, e o mesmo ocorre no caso do cimento autoadesivo. Já o jateamento com partículas de óxido de alumínio se mostrou eficaz, promovendo um aumento na resistência de união entre pino e cimento, sem a necessidade de qualquer tratamento adiciona (Prado et al., 2013). Para Conte et al., (2019) a clorexidina e o hipoclorito de sódio pode ser usados na cimentação adesiva dos pinos, sem que apresentem vantagens e/ou desvantagens na resistência de união, porém vão influenciar nos terços radiculares, podendo ser considerados inapropriados quanto à resistência de união no terço apical.

Tratando-se de cimentação de pinos intrarradiculares utilizando materiais do tipo resinoso, um bom resultado clínico deve-se, principalmente à ligação adesiva propriamente dita e também à retenção por atrito. Quanto à resistência, é possível afirmar que há uma relação direta da mesma com a fricção, onde a estabilidade da união da interface do sistema adesivo/dentina é associada à mesma, sendo assim, fica subentendido que se o pino é capaz de se manter em posição devido à fricção (Marques et al., 2014).

Para Marques et al., (2014), é coerente admitir como hipótese que quanto maior o vínculo de contato entre as paredes do canal e cimento resinoso, maior será a retenção, porém segundo Moro, Agostinho \& Matsumoto (2005) a ação de brocas utilizadas para remover o material obturador, produz uma nova "smearlayer" contendo cimento obturador e remanescentes de guta-percha plastificada, o que pode comprometer a resistência de união, pois os cimentos resinosos só são capazes de proporcionar alta resistência de união sob ótimas condições de limpeza dentinária.

\section{Conclusão}

Apesar do avanço nas técnicas e uma maior ampliação na criação de novos materiais, a literatura ainda não menciona qual pino pode ser considerado o melhor. Vários tipos de pinos pré-fabricados estão no mercado até hoje, sendo que, cada um possui indicações específicas. Quando utilizados de forma correta, ambos os pinos (núcleos metálicos fundidos e pinos de fibra de vidro) apresentam resultados satisfatórios, mas estudos feitos recentemente evidenciam que os pinos de fibra de vidro possuem uma resistência cada vez maior em relação a fraturas.

Já que as indicações e contraindicações dos retentores intrarradiculares foram apresentadas, estudos posteriores podem fazer comparações a respeito da resistência dos pinos aliados as diversas marcas que estão no mercado, com a 
finalidade de nortear os profissionais para os melhores materiais, além de especificar quais desses estão ligadas com a maior longevidade nas reabilitações em dentes anteriores e posteriores.

\section{Referências}

Andrioli, A. R. V., Coutinho, M., Vasconcelos, A. A. \& Miranda, M. E. (2016). Efeitos do reembasamento sobre a resistência ao cisalhamento por extrusão (push-out) de pinos de fibra de vidro. Rev. odontol. UNESP, 45(4). 227-233. https://doi.org/10.1590/1807-2577.14815

Carvalho, T. F., Frazão, A. R.; Dias Junior, L. C. de L., Castro, R. F. de, Klautau, E. B. \& Silva, J. M. da. (2019). Resistência à fratura de dentes tratados endodonticamente reforçados com pinos customizados de fibra de vidro e carbono. Dent. press endod., 9(1). 26-30. https://doi.org/10.14436/23582545.9.1.026-030.oar

Conte, T., Andrade, G. S., Gadonski, A, P., Santin, D. C. \& Naufel, F. S. (2019) Avaliação do efeito da clorexidina e do hipoclorito de sódio na cimentação adesiva de pinos - estudo in vitro. Rev Odontol UNESP. 48 (e) 20190020. http://dx.doi.org/10.1590/1807-2577.02019

Ferreira, M. B. C., Carlini, B., Jr., Sousa, Y. T. S., Gomes, E. A. \& Spazzin, A. O. (2018). Pino de fibra de vidro anatômico: relato de caso. Journal of oral investigations. 7(1). 51-61. https://doi.org/10.18256/2238-510X.2018.v7i1.2161

Finger, N. H., Paulus, M. \& Gazzoni, A. F. (2019). Potencial antimicrobiano de diferentes retentores intrarradiculares frente a Enterococcus faecalis: uma avaliação in vitro. Rev. Odontol UNESP. 48 (e)20190051. http://dx.doi.org/10.1590/1807-2577.05119

Franco, A. P. G. O., Portero, P. P., Martins, G. C., Calixto, A. L., Pereira, S. K., Gomes, J. C. \& Gomes, O. M. M. (2009). Pinos intrarradiculares estéticos caso clínico. Rev Inst Ciênc Saúde. 27(1).81-5. http://bases.bireme.br/cgi-bin/wxislind.exe/iah/online/?IsisScript=iah/iah.xis\&src=google\&base=LILACS \&lang=p\&nextAction=lnk\&exprSearch=545273\&indexSearch=ID.

Marques, J. N., Gonzalez, C. B., Silva, E. M., Pereira, G. D. S., Simão, R. A. \& Prado, M. (2016). Análise comparativa da resistência de união de um cimento convencional e um cimento autoadesivo após diferentes tratamentos na superfície de pinos de fibra de vidro. Rev Odontol UNESP. 45(2) 121-126. https://doi.org/10.1590/1807-2577.18615

Marques, V. F., Araújo, E. C. F., Silva, A. L. F., Tapety, C. M. C., Moreira, M. A. G. \& Casselli, D. S. M. (2014). Avaliação da resistência de união entre dentina e pinos de fibra de vidro utilizando três diferentes técnicas de cimentação. RFO UPF. 19(3) 283-287. https://doi.org/10.5335/rfo.v19i3.3864

Mazaro, J. V. Q., Santos, A. B., Zavanelli, A. C., Mello, C. C., Lemos, C. A. A. \& Filho, H. G. (2014). Avaliação dos fatores críticos para seleção e aplicação clínica dos pinos de fibra - relato de caso. Revista Odontológica de Araçatuba. 35(2). 26-36. https://repositorio.unesp.br/handle/11449/133254

Moro, M., Agostinho, A. M. \& Matsumoto, W. (2005). Núcleos Metálicos Fundidos X Pinos Pré-Fabricados. PCL - Revista Ibero-americana de Prótese Clínica e Laboratorial. 7(36). 167-172. https://www.dtscience.com/nucleos-metalicos-fundidos-x-pinos-pre-fabricados.

Oliveira, H. E., Ferreira, C. B., Graça, L. F. A., Bittencourt, R. C., Vallado, S. T. \& Gouvea, C. V. D. (2012). Distribuição das Tensões Relacionadas ao Uso de Retentores em Dentes Tratados Endodonticamente Utilizando o Método dos Elementos Finitos. Pesq Bras Odontoped Clin Integr. 12(1).41-46. https://doi.org/10.4034/PBOCI.2012.121.06

Pantoja, C. A. M. S., Pantoja, J. M. C. N., Ferraz, C. C. R. \& Almeida, J. F. A. (2011). Remoção de retentores metálicos intrarradiculares com o Saca-pinos M\&V: relato de casos clínicos. RPG Rev Pós Grad. 18(4). 260-265. http://www.fo.usp.br/?attachment_id=9603.

Pessoa, V. L. R., Monteiro, G. Q. de M.; Oliveira, N. G. de, Espíndola-Castro, L. F. (2019). Desgaste dentinário seletivo associado à pino de fibra de vidro: relato de caso. Rev. Ciênc. Plur, 5(3). 132-142. https://pesquisa.bvsalud.org/portal/resource/pt/biblio-1047965.

Prado, M. A. A., Kohl, J. C. M., Nogueira, R. D. \& Martins, V. R. G. (2014). Retentores Intrarradiculares: Revisão da Literatura. UNOPAR Cient Ciênc Biol Saúde. 16(1):51-55. https://doi.org/10.17921/2447-8938.2014v16n1p\%25p

Prado, N. A. S., Ferreira, R, S., Silva, T. C. A. P. \& Miranda, M. S. (2013). Influência da espessura do filme de cimento na resistência de união de pinos reforçados por fibras cimentados em canais radiculares. Rev Dental Press Estét. 10(1).89-95. https://br.clinicaldentistry.net/jcdr-v10n01-2013-89/.

Sá, T. C. M., Akaki, E., \& Sá, J, C, M. (2010). Pinos estéticos: qual o melhor sistema? Arquivo Brasileiro de Odontologia, 6(3), 179-184. http://periodicos.pucminas.br/index.php/Arquivobrasileirodontologia/article/view/2183.

Shiozawa, L. J., Capp, C. I., Mandetta, S., Cara, A. A., Tamaki, R. (2005). Retenção de pinos pré-fabricados e núcleos metálicos fundidos cimentados com cimento resinoso e fosfato de zinco. RPG Rev Pós Grad. 12(2) 248-254. https://pesquisa.bvsalud.org/portal/resource/pt/lil-457187.

Sigemori, R. M., Prieto, L. T., Oliveira, D. C. R. S., Coppini, E. K., Araújo, C. T. P., Dias, C. T. S., Paulillo, L. A. M. S. (2012). Reforço intrarradicular de raízes debilitadas. Rev. bras. odontol. 69(2) 250-254. http://revodonto.bvsalud.org/scielo.php?script=sci_arttext\&pid=S0034-72722012000200024.

Silva, R. V. C., Veronezi, M. C., Dekon, A. F. C., Silva, P. M. B., Silva, Silva, L. M., \& Andrade, A. M. (2009). Comparação da resistência à tração entre pinos metálicos (ni/cr) e de fibra de vidro cimentados com cimento resinoso. Salusvita. 28(1) 41-51. https://pesquisa.bvsalud.org/portal/resource/pt/lil-582291

Soares, D. N. S. \& Sant'Ana, L. L. P. (2018). Estudo Comparativo entre Pino de Fibra de Vidro e Pino Metálico Fundido: Uma Re visão de Literatura. Id on Line Rev. Mult. Psic. 12(42) 996-100. https://doi.org/10.14295/idonline.v12i42.1371 